\title{
Non invasive measurement of liver iron concentration using 3 Tesla magnetic resonance imaging: validation against biopsy
}

G. d'Assignies (MD, PhD) ${ }^{1,2}$, A Paisant (MD) ${ }^{1,3}$, E. Bardou-Jacquet (MD, PhD) ${ }^{3,4,5}$, A. Boulic $(\mathrm{MD})^{1}$, E. Bannier $(\mathrm{PhD})^{1,6}$, F. Lainé $(\mathrm{MD})^{3,4}, \mathrm{M}$. Ropert $(\mathrm{PhD})^{5,7}$, J. Morcet $(\mathrm{PhD})^{3}, \mathrm{H}$. SaintJalmes $(\mathrm{PhD})^{2,8}$, Y. Gandon (MD) ${ }^{1,2}$.

University Hospital of Rennes, France. 2 rue H. Le Guilloux, 35033 Rennes, France

(1) Department of Radiology, Rennes University Hospital, 2 rue H. Le Guilloux, 35033, Rennes, France

(2) LTSI, INSERM U1099, University of Rennes 1, Beaulieu Campus, 35042, Rennes, France

(3) Clinical investigation center, Rennes University Hospital, CIC INSERM 1414, 2 rue H. Le Guilloux, 35033, Rennes, France

(4) Department of Hepatology, Rennes University Hospital, 2 rue H. Le Guilloux, 35033, Rennes, France

(5) INSERM UMR991, Rennes University Hospital, 2 rue H. Le Guilloux, 35033, Rennes, France

(6) VisAGeS U746 Unit/Project, INSERM/INRIA, IRISA, UMR CNRS 6074, University of Rennes 1, Beaulieu Campus, 35042, Rennes, France

(7) Department of Biochemistry, Rennes University Hospital, 2 rue H. Le Guilloux, 35033, Rennes, France

(8) CRLCC, Centre Eugène Marquis, Rennes, F-35000, France 
Corresponding author: Yves Gandon

Address: Unité d'imagerie abdominale, Service de Radiologie, Hôpital Pontchaillou, CHU

Rennes, 2 rue H. Le Guilloux, 35033 Rennes, France

Email: yves.gandon@chu-rennes.fr

Telephone: $\quad+33(0) 2.99 .28 .24 .39$

Fax: $+33(0) 2.99 .28 .43 .64$

\section{ABSTRACT}

OBJECTIVES: To evaluate the performance and limitations of the R2* and signal intensity ratio (SIR) methods for quantifying liver iron concentration (LIC) at 3T.

METHODS: One hundred and five patients who underwent a liver biopsy with biochemical LIC $\left(\right.$ LIC $\left._{\mathrm{b}}\right)$ were included prospectively. A 3T MRI scan with a breath-hold multiple-echo gradient-echo sequence (mGRE) was undertaken for all patients. LIC calculated by 3T SIR algorithm (LICSIR) and by $\mathrm{R} 2 *\left(\mathrm{LIC}_{\mathrm{R} 2 *}\right)$ were correlated to $\mathrm{LIC}_{\mathrm{b}}$. Sensitivity and specificity were calculated. The comparison of methods was analyzed for successive classes.

RESULTS: $\mathrm{LIC}_{\mathrm{b}}$ was strongly correlated to $\mathrm{R} 2 *(\mathrm{r}=0.95 \mathrm{p}<0.001)$ and $\mathrm{LIC}_{\mathrm{SIR}}(\mathrm{r}=0.92 \mathrm{p}<0.001)$. In comparison to $\mathrm{LIC}_{\mathrm{b}}, \mathrm{LIC}_{\mathrm{R} 2 *}$ and $\mathrm{LIC}_{\mathrm{SIR}}$ detect liver iron overload with a sensitivity/specificity of $0.96 / 0.93$ and $0.92 / 0.95$, respectively and a bias \pm SD of $7.6 \pm 73.4$ and $14.8 \pm 37.6 \mu \mathrm{mol} / \mathrm{g}$, respectively. $\mathrm{LIC}_{\mathrm{R} 2 *}$ presented the lowest differences for patients with $\mathrm{LIC}_{\mathrm{b}}$ values under $130 \mu \mathrm{mol} / \mathrm{g}$. Above this value, $\mathrm{LIC}_{\text {SIR }}$ has the lowest differences.

CONCLUSIONS: At 3T, R2* provides precise LIC quantification for lower overload but the SIR method is recommended to overcome R2* limitations in higher overload. Our software, available on mrquantif.org, uses jointly both methods and selects the best one. 


\section{KEYWORDS}

Iron, Liver, Magnetic resonance imaging, Hemosiderosis, Non-alcoholic fatty liver disease, Hemochromatosis.

\section{KEY POINTS}

- Liver iron can be accurately quantified by MRI at $3 \mathrm{~T}$

- At 3T, R2* provides precise quantification of slight liver iron overload

- At 3T, SIR method is recommended in case of high iron overload

- Slight liver iron overload present in metabolic syndrome can be depicted.

- Treatment can be monitored with great confidence.

\section{ABBREVIATIONS}

SIR: Signal intensity ratio

LIC: liver iron concentration

LIC $_{\mathrm{b}}$ : LIC assessed by biopsy using biochemical analysis

LIC SIR: LIC calculated by SIR method

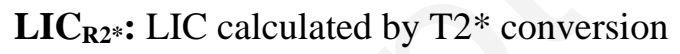

mGRE: Multiple-echo gradient-echo sequence

NASH: Non-alcoholic steatohepatitis

DIOS: Dysmetabolic iron overload syndrome

MRI: Magnetic resonance imaging

BMI: Body mass index

AUC: Area under the curve 


\section{Introduction}

Liver iron content (LIC) is a surrogate marker of whole-body iron load. In overload diseases such as primary or secondary hemochromatosis, LIC measurement is mandatory for guiding therapeutic decisions. Liver iron overload may also be present in non-alcoholic steatohepatitis (NASH) and dysmetabolic iron overload syndrome (DIOS), which are both highly prevalent in the Western population (1). The main complications are cirrhosis and hepatocellular carcinoma. Many studies $(2,3)$ have suggested a close correlation between iron deposition and carcinogenesis.

The gold-standard method for detecting and quantifying liver iron overload is histopathological analysis of a liver sample collected by biopsy with biochemical analysis of the core fragment. The biopsy procedure is both invasive and painful and carries some risk of complications (4). In addition, the very small liver sample may not be representative of the whole liver in cases of heterogeneous iron distribution (5).

Non-invasive, quantitative assessment of LIC by $1.5 \mathrm{~T}$ magnetic resonance imaging (MRI) has been extensively validated against histology by calculating the relaxation rates R2 and R2* (6-11) and/or the signal intensity ratio (SIR) between the liver and paraspinal muscles (12-14). MRI is thus now used in routine clinical practice to diagnose, quantify and monitor iron overload (15).

In recent years, 3T MRI has become more widespread. In view of the shift in magnetic field strength, acquisition parameters need to be adapted and new reference values proposed.

Better sensitivity and accuracy can be expected at 3T, improving diagnosis of DIOS with low iron burden. Conversely, quantification of high overload cases may prove more difficult (16).

Recently, the SIR method, based on several single-echo GRE sequences, has been validated against histology at 3T (17). 
The purpose of our study was to evaluate the ability of the R2* method to detect and quantify liver iron at 3T using biochemical quantification as the reference method. Our secondary goal was to compare, at the 3T field strength, two major LIC quantification methods: R2* and SIR.

\section{Materials and Methods}

\section{Patient population}

Between January 2007 and January 2013, all patients referred for liver biopsy and in whom liver iron overload was suspected according to their disease were prospectively recruited. All patients provided written informed consent to participate in this prospective single-center clinical trial. In addition to usual care, an MRI scan was scheduled to assess hepatic iron stores. Age, sex and body mass index were recorded.

\section{Biochemical liver iron concentration}

Liver biopsy was indicated as per the guidelines of the American Association for the Study of Liver Diseases $(18,19)$. A biopsy sample was taken from the right lobe of the liver using a 16 gauge needle (Hepafix 16G, Braun, Melsingen, Germany) under ultrasound guidance. Biochemical liver iron concentration $\left(\mathrm{LIC}_{\mathrm{b}}\right)$ was measured using Barry and Sherlock's method for biopsy samples taken from paraffin-embedded blocks (20). Liver iron overload was defined as an $\mathrm{LIC}_{\mathrm{b}}$ greater than $35 \mu \mathrm{mol} / \mathrm{g}$ (dry liver). Biochemical analysis was blinded to MRI results.

\section{Magnetic resonance imaging protocol}

The study was performed with two 3T MRI scanners: first with Achieva (Philips, Best, Netherlands) and then with Magnetom Verio (Siemens Healthcare, Erlangen, Germany). The body coil was used as the receive coil to achieve homogeneous signal intensity in the imaged section and avoid signal depth fall-off. Only the Siemens scanner had a compensation method for better B1 homogeneity. There was a slight difference in resonance frequency (127.79 vs. $123.24 \mathrm{MHz}$ ) between the two scanners. Using the body coil, one multi-echo gradient echo (mGRE) sequence was performed, with 11 echoes. The selected TEs were slightly different depending on the scanner: a multiple of $1.15 \mathrm{~ms}$ for the Philips group and $1.23 \mathrm{~ms}$ for the Siemens group. Pixel bandwidth was $1161 \mathrm{~Hz}$ for the Philips group and 
$1048 \mathrm{~Hz}$ for the Siemens group. The remaining parameters were identical for both machines: 400x400mm ${ }^{2}$ field of view; 128*121 acquisition matrix; 256x256 reconstruction matrix with a pixel size of $1.56 \times 1.56 \mathrm{~mm}^{2} ; 120 \mathrm{~ms}$ repetition time; $20^{\circ}$ flip angle; $7 \mathrm{~mm}$ slice thickness; 1 excitation. The breath-hold acquisition lasted 15s.

\section{MRI data analysis}

Measurements were conducted using an in-house Java program integrating ImageJ functions (NIH, Bethesda, USA). All data were analyzed by a radiologist (with 10 years' experience in abdominal radiology) who was blind to clinical information and to the biopsy result.

On the selected slice, 3 ROIs with a diameter of $2.5 \mathrm{~cm}\left(4.9 \mathrm{~cm}^{2}\right)$ were placed in the right liver area, taking care to avoid large vessels, biliary tracts, parenchymatous lesions and artifacts, 2 ROIs with a diameter of $2 \mathrm{~cm}\left(3.1 \mathrm{~cm}^{2}\right)$ in the right and left paraspinal muscles and $1 \mathrm{ROI}$ with a diameter of $3 \mathrm{~cm}$ $\left(7.1 \mathrm{~cm}^{2}\right)$ in the air outside of the body for noise measurement. All ROIs were automatically copied to the same place on each echo of this selected location. The placement of the ROIs is illustrated in Figure 1.

Before performing fitting, we applied a noise subtraction algorithm to subtract the mean background noise from the liver signal. Then $\mathrm{T} 2 *$ values were automatically calculated using a simplex non-linear algorithm to fit the magnitude of the complex signal from all echoes or only from in-phase echoes when the signal of the first out-of-phase echo was lower than the signal of the first in-phase echo.

Thus $\mathrm{T} 2 *$ was calculated according to the formula: Liver signal - noise $=M 0 \cdot e^{-\left(\frac{T E}{T 2 *}\right)}$

$\mathrm{R} 2 *$ was calculated as follows: $\mathrm{R} 2^{*}=1 / \mathrm{T} 2^{*}$, and we used the linear correlation with $\mathrm{LIC}_{\mathrm{b}}$ to determine $\mathrm{LIC}_{\mathrm{R} 2 *}$.

The liver-to-muscle signal intensity ratio (SIR) method was used to calculate LIC $_{\text {SIR }}$ with the algorithm derived from the same patient series using 5 single-echo GRE sequences (17). Only the first

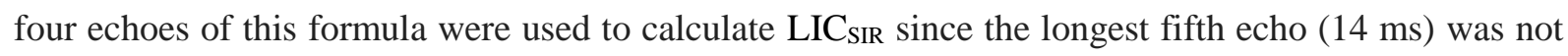
obtained in the mGRE acquisition. 


\section{Statistical analysis}

Statistical analyses were performed using SAS 9.4 (SAS Institute, Cary, NC).

Qualitative variables were expressed as numbers and percentages. Quantitative data were expressed as means \pm standard deviations (SD) if normally distributed and medians (Q1-Q3) if not normally distributed.

Given that LIC quantification variables were not normally distributed, we calculated non-linear correlation coefficients (Spearman) to estimate the strength of the linear relationship between $\mathrm{LIC}_{\mathrm{R} 2 *}$ or $\mathrm{LIC}_{\mathrm{SIR}}$ and $\mathrm{LIC}_{\mathrm{b}}$.

Similarly, in order to compare measurements using the Philips or Siemens scanner, Generalized Poisson Mixed Models (GLIMMIX procedure) were used with or without adjustment for sex, BMI and age.

Agreement between LIC quantifications was assessed using the Bland and Altman method, calculating the mean difference (estimated bias, $\mathrm{d}$ ), the standard deviation of the differences (precision, SD), and the limits of agreement $(\mathrm{d} \pm 1.96 \mathrm{SD})$. Student's t-test was used to determine whether the bias between measurement methods was significant.

Optimal cut-off values for the threshold of $\mathrm{LIC}_{\mathrm{b}}$ at $36 \mu \mathrm{mol} / \mathrm{g}$ were obtained by optimization of the Youden index from AUROC curve analysis.

The area under the curve, sensitivity, specificity, positive and negative predictive values were calculated for both $\mathrm{LIC}_{\mathrm{R} 2 *}$ and $\mathrm{LIC}_{\mathrm{SIR}}$.

In order to compare the two methods at different levels, the cohort was divided into equal successive classes according to the values of $\mathrm{LIC}_{\mathrm{b}}$. Then, $\mathrm{LIC}_{\mathrm{b}}-\mathrm{LIC}_{\mathrm{R} 2 *}$ and $\mathrm{LIC}_{\mathrm{b}}-\mathrm{LIC}_{\mathrm{SIR}}$ were calculated and compared at the different $\mathrm{LIC}_{\mathrm{b}}$ levels. A similar comparison, corresponding more to the practical intent to diagnose, was also done by using $\mathrm{LIC}_{\mathrm{R} 2 * \text { classes. }}$

$P$ values less than 0.05 were considered statistically significant.

\section{RESULTS}

\section{Patient population}

One hundred and five patients were prospectively included between January 2007 and January 2013 (Figure 2). Fifty-eight (55\%) had hyperferritinemia, 52 (50\%) had metabolic syndrome, 15 (14\%) had 
chronic alcohol intoxication, $15(14 \%)$ had either hepatitis B or C, and $6(6 \%)$ had other liver diseases (autoimmune hepatitis, primary biliary cirrhosis).

The first 36 patients (34\%) included were scanned with the Philips scanner and the following 69 $(66 \%)$ with the Siemens scanner. Mean $( \pm \mathrm{SD})$ age was $55.8 \pm 12.7$ and $50 \pm 12.8$ years for women and men, respectively $(\mathrm{p}=0.03)$. One hundred and one patients underwent the MRI examination and the biopsy on the same day while 4 patients experienced an interval between the biopsy and MRI of less than 15 days. The $\mathrm{LIC}_{\mathrm{b}}$ concentration ranged from 0 to $630 \mu \mathrm{mol} / \mathrm{g}$, and 49 patients (47\%) had normal LIC $_{b}$ values $<36 \mu \mathrm{mol} / \mathrm{g}$. Fifty six patients had a liver iron overload. It was due to genetic hemochromatosis in 31 patients $\left(\mathrm{LIC}_{\mathrm{b}}\right.$ mean $=286 \pm 148 \mu \mathrm{mol} / \mathrm{g}$, range $=43-630 \mu \mathrm{mol} / \mathrm{g}$ ), to dysmetabolic syndrom in 22 patients $\left(\mathrm{LIC}_{\mathrm{b}}\right.$ mean $=62 \pm 27 \mu \mathrm{mol} / \mathrm{g}$, range $=36-123 \mu \mathrm{mol} / \mathrm{g}$ ) and to other causes (alcoholic or viral hepatitis) in 3 patients $\left(\mathrm{LIC}_{\mathrm{b}}\right.$ mean $=42 \pm 5 \mu \mathrm{mol} / \mathrm{g}$, range $=36-46 \mu \mathrm{mol} / \mathrm{g}$ ). Patient characteristics are provided in Table 1.

Our analysis without / with the adjustment for BMI, sex and age yielded no difference in the distribution of $\mathrm{LIC}_{\mathrm{b}}(\mathrm{p}=0.65 / \mathrm{p}=0.19), \operatorname{LIC}_{\mathrm{R} 2^{*}}(\mathrm{p}=0.49 / \mathrm{p}=0.14)$ and $\mathrm{LIC}_{\mathrm{SIR}}(\mathrm{p}=0.50 / 0.27)$ results between the two groups using MRI machines from different manufacturers.

\section{$R 2 *$ and $L I C_{R 2 *}$ measurements}

Linear regression between $\mathrm{LIC}_{\mathrm{b}}$ and $\mathrm{R} 2 *$ is shown in Figure $3 \mathrm{a}$ and yielded the following equation:

$$
\mathrm{LIC}_{\mathrm{R} 2 *}(\mu \mathrm{mol} / \mathrm{g})=0.316 \mathrm{R} 2 *+7.6
$$

The Spearman correlation coefficient $(r=0.95 \mathrm{p}<0.001)$ indicates a strong positive correlation between $\mathrm{LIC}_{\mathrm{b}}$ and $\mathrm{R} 2 *$.

Figure $3 \mathrm{~b}$ shows the Bland-Altman plot of the difference vs. mean values of $\mathrm{LIC}_{\mathrm{b}}$ and $\mathrm{LIC}_{\mathrm{R} 2}$ * measurements. The bias (SD) or average difference between the results of the two methods was 7.6 (73.4) $\mu \mathrm{mol} / \mathrm{g}$ and the $95 \%$ limits of agreement were $-136.4 \mu \mathrm{mol} / \mathrm{g}$ and $151.5 \mu \mathrm{mol} / \mathrm{g}$. The bias was not statistically significantly different to zero $(\mathrm{p}=0.74)$.

With the reference threshold established at $\mathrm{LIC}_{\mathrm{b}}=36 \mu \mathrm{mol} / \mathrm{g}$, ROC curves obtained with $\mathrm{LIC}_{\mathrm{R} 2 * \text { results }}$ 
showed an area under the curve (AUC) of 0.987 . The best threshold was given for $\mathrm{LIC}_{\mathrm{R}^{*}}$ at $32 \mu \mathrm{mol} / \mathrm{g}$, corresponding to an $\mathrm{R} 2 *$ of $77 \mathrm{~s}^{-1}$, and a T2* of $13 \mathrm{~ms}$, with 47 true positives, 4 false positives, 52 true negatives and 2 false negatives. The sensitivity was 0.96 (95\% CI: $0.9 ; 1.01)$ and the specificity 0.93 (95\% CI: $0.86 ; 1.0)$.

Taking in consideration only the 76 patients with $\mathrm{LIC}_{\mathrm{b}}$ below $130 \mu \mathrm{mol} / \mathrm{g}$, linear regression yielded the following equation:

$$
\mathrm{LIC}_{\mathrm{R} 2 *}(\mu \mathrm{mol} / \mathrm{g})=0.314 \mathrm{R} 2 *-0.96
$$

The best threshold was then given for $\mathrm{LIC}_{\mathrm{R} 2 *}$ at $27 \mu \mathrm{mol} / \mathrm{g}$, corresponding to an $\mathrm{R} 2 *$ of $89 \mathrm{~s}^{-1}$, and a T2* of $11 \mathrm{~ms}$, with 44 true positives, 2 false positives, 25 true negatives and 5 false negatives. The sensitivity was 0.90 (95\% CI: $0.81 ; 0.98)$ and the specificity 0.93 (95\% CI: $0.83 ; 1.0$ ).

\section{LIC SIR measurement}

Linear regression between $\mathrm{LIC}_{\mathrm{b}}$ and $\mathrm{LIC}_{\mathrm{SIR}}$ is shown in Figure 3c. The Spearman correlation coefficient $(r=0.92 \mathrm{p}<0.001)$ indicates a strong positive correlation between $\mathrm{LIC}_{\mathrm{SIR}}$ and $\mathrm{LIC}_{\mathrm{b}}$. Figure 3d shows the Bland-Altman plot of the difference vs. mean values of $\mathrm{LIC}_{\mathrm{b}}$ and $\mathrm{LIC}_{\mathrm{SIR}}$ measurements. The bias (SD) or average difference between the results of the two methods was 14.8 (37.6) and the $95 \%$ limits of agreement were -59.0 and $88.5 \mu \mathrm{mol} / \mathrm{g}$. The bias was statistically significantly different to zero $(\mathrm{p}<0.0001)$.

With the reference threshold established at $\mathrm{LIC}_{\mathrm{b}}=36 \mu \mathrm{mol} / \mathrm{g}$, the ROC curves obtained with $\mathrm{LIC}_{\text {SIR }}$ results showed an AUC of 0.965 . The best threshold was given for $\mathrm{LIC}_{\mathrm{SIR}}=20 \mu \mathrm{mol} / \mathrm{g}$ with 45 true positives, 3 false positives, 53 true negatives and 4 false negatives. The sensitivity was 0.92 (95\% CI: $0.84 ; 0.99)$ and the specificity 0.95 (95\% CI: $089 ; 1.0)$.

\section{Comparison between $\mathrm{LIC}_{R 2^{*}}$ and $L I C_{S I R}$ measurements}

The Spearman correlation coefficient $(r=0.95 \mathrm{p}<0.001)$ indicates a strong positive correlation between $\mathrm{LIC}_{\mathrm{R} 2 *}$ and $\mathrm{LIC}_{\mathrm{SIR}}$. Figure 4 shows the mean differences (absolute values) between $\mathrm{LIC}_{\mathrm{SIR}}$ and $\mathrm{LIC}_{\mathrm{b}}$ or between $\mathrm{LIC}_{\mathrm{R} 2^{*}}$ and $\mathrm{LIC}_{\mathrm{b}}$ according to $\mathrm{LIC}_{\mathrm{b}}$ or $\mathrm{LIC}_{\mathrm{R} 2^{*}}$ class. With $\mathrm{LIC}_{\mathrm{b}}$ classes, $\mathrm{LIC}_{\mathrm{R} 2^{*}}$ 
presented the lowest differences for patients with $\mathrm{LIC}_{\mathrm{b}}$ values under $130 \mu \mathrm{mol} / \mathrm{g}$ and the highest differences for patients above $190 \mu \mathrm{mol} / \mathrm{g}$ (Figure 4a). Using $\mathrm{LIC}_{\mathrm{R} 2 *}$ classes, the differences increased above $130 \mu \mathrm{mol} / \mathrm{g}$ (Figure 4b).

\section{DISCUSSION}

With a shortest TE of $1.2 \mathrm{~ms}$, liver iron overload can be reliably quantified by MRI at $3 \mathrm{~T}$ with the R2* for patients with biopsy-proven LIC under $130 \mu \mathrm{mol} / \mathrm{g}$ but the SIR method appears more robust for higher iron overload.

The R2* calculation is well known and its clinical use is well established at $1.5 \mathrm{~T}$. In the literature, there are 5 main publications, validating R2* against LIC determined by biopsy $(6,8-11)$. Conversion formulas have been proposed to estimate LIC from R2* $\left(\mathrm{s}^{-1}\right)$ with a slope of 0.025 to 0.032 to obtain the LIC value in $\mathrm{mg} / \mathrm{g}$. Pooling the data from the main publications, Henninger found a mean slope of 0.029 (11). Then, to obtain the LIC in $\mu \mathrm{mol}$ instead of $\mathrm{mg} / \mathrm{g}$, we multiplied this mean slope by 18 to obtain 0.52 . So, at $1.5 \mathrm{~T}$, simply by dividing by 2 the value of $\mathrm{R} 2 *$ expressed in $\mathrm{s}^{-1}$ we have a correct approximation of LIC expressed in $\mu \mathrm{mol} / \mathrm{g}$.

No such validation with biopsies has been done at 3T. Theoretical calculations suggest a doubling of R2* from 1.5 to $3 \mathrm{~T}$ (21). Then the mean slope to obtain the LIC value in $\mu \mathrm{mol} / \mathrm{g}$ should be divided by 2 and should be approximately 0.26 . Anwar's (22) results in 5 patients seem to confirm this hypothesis but with significant delay between MRI and biopsy. However, in our series we obtained a slope of 0.316 , slightly higher than the slope expected by extrapolation of $1.5 \mathrm{~T}$ polled data but close to half the higher slope proposed at $1.5 \mathrm{~T}$ by Garbowski, who used the same laboratory reference (10). In our series, the background noise subtraction leads to a higher value of $\mathrm{R} 2 *$ and partly explains the residual difference with Garbowski's results. This emphasizes the need for a standardized protocol to obtain more comparable results. 
The higher magnetic susceptibility observed at $3 \mathrm{~T}$ introduced limitations in the R2* calculation. For high overloads, there is a strong decrease in liver signal intensity. It is then difficult to obtain a correct exponential curve fit.

The SIR method is also widely recognized and used for hepatic iron quantification at $1.5 \mathrm{~T}$. Our study evaluated this method at $3 \mathrm{~T}$ using the algorithm defined from single-echo sequences (17). The results we obtained with an mGRE sequence showed good correlations but with a slight overall overestimation and a slight underestimation for low values because the longest TE, around $14 \mathrm{~ms}$, was not included in the mGRE sequence. For slight to moderate overloads, below $130 \mu \mathrm{mol} / \mathrm{g}$, almost exclusively patients with DIOS, our study showed a better correlation of the R2* method than the SIR method to $\mathrm{LIC}_{\mathrm{b}}$. However, in patients with high $\mathrm{LIC}_{\mathrm{b}}$ above $130 \mu \mathrm{mol} / \mathrm{g}$, corresponding exclusively, in our study, to patient with genetic hemochromatosis, the SIR method provides a better correlation to $\mathrm{LIC}_{\mathrm{b}}$. At $1.5 \mathrm{~T}$, quantification was possible by SIR up to $350 \mu \mathrm{mol} / \mathrm{g}$ by using the shortest in-phase TE of $4 \mathrm{~ms}$. Rose overcomes this limit by using a shorter first TE of $1.8 \mathrm{~ms}$ (23). At 3T, a first TE of $1.2 \mathrm{~ms}$ is short enough to give a liver signal over the signal noise and to allow a SIR estimation in high overload.

Our study is the largest series calibrating $\mathrm{R} 2 *$ versus $\mathrm{LIC}_{\mathrm{b}}$, for any magnetic field strength. It validates the use of 3T MRI for hepatic iron quantification. In comparison to the biopsy with biochemical determination of iron, we propose a formula to convert $\mathrm{R} 2 *$ at $3 \mathrm{~T}$ to $\mathrm{LIC}_{\mathrm{b}}$. Despite variation in technical characteristics, there was no significant difference between the two machines used. Although the use of 3T MRI is becoming more widespread, some centers only have a 3T magnetic field for abdominal imaging. There is a strong need for reference values at 3T. Moreover, the use of a 3T magnetic field allows for more accurate quantification of slight to moderate overloads. Improving sensitivity is clinically relevant regarding the increasing incidence of DIOS with low iron overload.

Our study has certain limitations. First, the shortest TE was about $1.2 \mathrm{~ms}$, a value which is also the first TE usually proposed by MR vendors in most built-in protocols dedicated to hepatic iron and fat quantification. Obviously, this TE is not short enough at $3 \mathrm{~T}$ to correctly calculate R2* in the case of 
high overload. It is technically difficult to use a first TE of $0.4 \mathrm{~ms}$, which is half the shortest TE of $0.8 \mathrm{~ms}$ proposed by Wood at $1.5 \mathrm{~T}$ (8). Very short TEs will be available using ultrashort echo time (UTE) imaging (24). In the meantime, the main risk is not being unable to quantify correctly a high overload, which has only a small impact on patient management, but miscalculating R2* and hence underestimating liver iron overload. For example a patient with an R2* of $512 \mathrm{~s}^{-1}$, corresponding to an $\mathrm{LIC}_{\mathrm{R}^{*}}$ of $130 \mu \mathrm{mol} / \mathrm{g}$, actually had an $\mathrm{LIC}_{\mathrm{b}}$ of $480 \mu \mathrm{mol} / \mathrm{g}$. This type of error explains how the difference between $\mathrm{LIC}_{\mathrm{R} 2 *}$ and $\mathrm{LIC}_{\mathrm{b}}$ increases faster with $\mathrm{LIC}_{\mathrm{R} 2 *}$ classes than with $\mathrm{LIC}_{\mathrm{b}}$ classes. So, to overcome this limitation, we propose either greatly reducing the shortest TE or combining both SIR and T2* methods. Second, we used two different machines with a slight magnetic field difference (3\%). Acquisition parameters were as close as possible. However, there were also slight TE differences (8\%). This could have produced errors particularly for the SIR method which does not take into account TE differences between the two units. The absence of B1 heterogeneity correction with the first machine may also lead, in some cases, to an overestimation of $\mathrm{LIC}_{\mathrm{SIR}}$ through reduction of the paraspinal muscle signal, as described with single-echo sequences (17). Third, we used the body coil for both methods. This coil is necessary for the SIR method. A surface coil allows a higher signal for $\mathrm{R} 2 *$ calculation but this is offset by larger voxels $\left(17 \mathrm{~mm}^{3}\right)$ and $\mathrm{T} 2 *$ fitting to the entire ROI instead of producing a pixel-wise map. Fourth, we only use 4 of the 5 echoes used by the 3T SIR algorithm based on single-echo sequences. This explains the bias observed for the low values of $\mathrm{LIC}_{\mathrm{b}}$ with a LIC $_{\text {SIR }}$ cut-off of $20 \mu \mathrm{mol} / \mathrm{g}$ for determining overloaded patients. A new version of the algorithm taking into account the reduction in the number of echoes obtained has now been incorporated into our dedicated software. Nevertheless, this has no practical impact since at that level of overload R2* is the most precise method.

This study validates hepatic iron quantification by MRI at $3 \mathrm{~T}$, with a conversion formula to $\mathrm{LIC}_{\mathrm{b}}$ obtained from biopsy material. With the selected TEs, the R2* method is more accurate for slight to moderate hepatic iron overload whereas the SIR method is more accurate for high overloads. Shorter TEs are needed to improve performance for quantifying massive iron overload by R2* [24]. In the meantime, both methods should be used simultaneously with a breath-hold mGRE sequence acquired 
using the body coil. The sequence protocol we propose can be applied to the majority of MRI scanners without the need to purchase a specific option. Detailed sequence parameters and a dedicated DICOM software program, incorporating both calculations with cross-checks, are available at www.mrquantif.org 
1. Blachier M, Leleu H, Peck-Radosavljevic M, et al (2013) The burden of liver disease in Europe: a review of available epidemiological data. J Hepatol 58:593-608. doi: 10.1016/j.jhep.2012.12.005

2. Moukhadder HM, Halawi R, Cappellini MD, Taher AT (2017) Hepatocellular carcinoma as an emerging morbidity in the thalassemia syndromes: A comprehensive review. Cancer 123:751758. doi: $10.1002 /$ cncr.30462

3. Deugnier Y, Turlin B (2011) Pathology of hepatic iron overload. Semin Liver Dis 31:260-271. doi: $10.1055 / \mathrm{s}-0031-1286057$

4. Hatfield MK, Beres RA, Sane SS, Zaleski GX (2008) Percutaneous imaging-guided solid organ core needle biopsy: coaxial versus noncoaxial method. AJR Am J Roentgenol 190:413-417. doi: 10.2214/AJR.07.2676

5. Wood JC, Zhang P, Rienhoff H, et al (2015) Liver MRI is more precise than liver biopsy for assessing total body iron balance: a comparison of MRI relaxometry with simulated liver biopsy results. Magn Reson Imaging 33:761-767. doi: 10.1016/j.mri.2015.02.016

6. Anderson LJ, Holden S, Davis B, et al (2001) Cardiovascular T2-star (T2*) magnetic resonance for the early diagnosis of myocardial iron overload. Eur Heart J 22:2171-2179.

7. St Pierre TG, Clark PR, Chua-anusorn W, et al (2005) Noninvasive measurement and imaging of liver iron concentrations using proton magnetic resonance. Blood 105:855-861. doi: 10.1182/blood-2004-01-0177

8. Wood JC, Enriquez C, Ghugre N, et al (2005) MRI R2 and R2* mapping accurately estimates hepatic iron concentration in transfusion-dependent thalassemia and sickle cell disease patients. Blood 106:1460-1465. doi: 10.1182/blood-2004-10-3982

9. Hankins JS, McCarville MB, Loeffler RB, et al (2009) R2* magnetic resonance imaging of the liver in patients with iron overload. Blood 113:4853-4855. doi: 10.1182/blood-2008-12-191643

10. Garbowski MW, Carpenter J-P, Smith G, et al (2014) Biopsy-based calibration of T2* magnetic resonance for estimation of liver iron concentration and comparison with R2 Ferriscan. J Cardiovasc Magn Reson Off J Soc Cardiovasc Magn Reson 16:40. doi: 10.1186/1532-429X-1640

11. Henninger B, Zoller H, Rauch S, et al (2015) R2* relaxometry for the quantification of hepatic iron overload: biopsy-based calibration and comparison with the literature. RöFo Fortschritte Auf Dem Geb Röntgenstrahlen Nukl 187:472-479. doi: 10.1055/s-0034-1399318

12. Gandon Y, Olivié D, Guyader D, et al (2004) Non-invasive assessment of hepatic iron stores by MRI. Lancet 363:357-362. doi: 10.1016/S0140-6736(04)15436-6

13. Alústiza JM, Artetxe J, Castiella A, et al (2004) MR quantification of hepatic iron concentration. Radiology 230:479-484. doi: 10.1148/radiol.2302020820 
14. Ernst O, Rose C, Sergent G, L'Herminé C (1999) Hepatic iron overload: quantification with MR imaging at 1.5 T. AJR Am J Roentgenol 172:1141-1142. doi: 10.2214/ajr.172.4.10587170

15. Brissot P, Troadec M-B, Bardou-Jacquet E, et al (2008) Current approach to hemochromatosis. Blood Rev 22:195-210. doi: 10.1016/j.blre.2008.03.001

16. Meloni A, Positano V, Keilberg P, et al (2012) Feasibility, reproducibility, and reliability for the $\mathrm{T} * 2$ iron evaluation at $3 \mathrm{~T}$ in comparison with 1.5 T. Magn Reson Med Off J Soc Magn Reson Med Soc Magn Reson Med 68:543-551. doi: 10.1002/mrm.23236

17. Paisant A, Boulic A, Bardou-Jacquet E, et al (2017) Assessment of liver iron overload by $3 \mathrm{~T}$ MRI. Abdom Radiol N Y 42:1713-1720. doi: 10.1007/s00261-017-1077-8

18. Rockey DC, Caldwell SH, Goodman ZD, et al (2009) Liver biopsy. Hepatol Baltim Md 49:1017-1044. doi: 10.1002/hep.22742

19. Bacon BR, Adams PC, Kowdley KV, et al (2011) Diagnosis and management of hemochromatosis: 2011 practice guideline by the American Association for the Study of Liver Diseases. Hepatol Baltim Md 54:328-343. doi: 10.1002/hep.24330

20. Barry M, Sherlock S (1971) Measurement of liver-iron concentration in needle-biopsy specimens. Lancet 1:100-103.

21. Storey P, Thompson AA, Carqueville CL, et al (2007) R2* imaging of transfusional iron burden at $3 \mathrm{~T}$ and comparison with 1.5T. J Magn Reson Imaging JMRI 25:540-547. doi: 10.1002/jmri.20816

22. Anwar M, Wood J, Manwani D, et al (2013) Hepatic Iron Quantification on 3 Tesla (3 T) Magnetic Resonance (MR): Technical Challenges and Solutions. Radiol Res Pract 2013:628150. doi: $10.1155 / 2013 / 628150$

23. Rose C, Vandevenne P, Bourgeois E, et al (2006) Liver iron content assessment by routine and simple magnetic resonance imaging procedure in highly transfused patients. Eur J Haematol 77:145-149. doi: 10.1111/j.0902-4441.2006.t01-1-EJH2571.x

24. Krafft AJ, Loeffler RB, Song R, et al (2017) Quantitative ultrashort echo time imaging for assessment of massive iron overload at 1.5 and 3 Tesla. Magn Reson Med. doi: $10.1002 / \mathrm{mrm} .26592$ 


\section{TABLES}

Table 1: Patient characteristics $(n=105)$

\begin{tabular}{ll}
\hline Male/Female & $\mathbf{6 8 / 3 7}$ \\
\hline Mean age (SD) & $\mathbf{5 2 . 1}(+/-\mathbf{1 3})$ \\
\hline MRI scanner manufacturer (Siemens/Philips) & $\mathbf{6 9 / 3 6}$ \\
\hline Body Mass Index, mean (SD) & $\mathbf{2 7 . 8 ( 4 . 2 )}$ \\
\hline LIC ${ }_{\mathrm{b}} \mu \mathrm{mol} / \mathrm{g}$, median [interquartile range] & $\mathbf{3 7 . 5}[\mathbf{1 8 . 2}-\mathbf{1 3 0 . 1}]$ \\
\hline $\mathrm{LIC}_{\mathrm{SIR}} \mu \mathrm{mol} / \mathrm{g}$, median [interquartile range] & $\mathbf{3 0}[\mathbf{0}-\mathbf{1 2 0}]$ \\
\hline $\mathrm{LIC}_{\mathrm{R} 2 *} \mu \mathrm{mol} / \mathrm{g}$, median [interquartile range] & $\mathbf{3 2 . 9}[\mathbf{1 8 . 5 - 1 1 7 . 7 ]}$ \\
\hline
\end{tabular}




\section{FIGURE CAPTIONS}

Figure 1: Screen-copy of the in-house software viewer with example of ROIs placement.

Figure 2: Flow chart.

Figure 3: Comparison between $\mathrm{LICb}$ and $\mathrm{R} 2 *$ or $\mathrm{LIC}_{\text {SIR }}$ : a) Linear regression between $\mathrm{R} 2 *$ and $\mathrm{LIC}_{\mathrm{b}}$, b) Bland-Altman plot of the difference vs. average of $\mathrm{LIC}_{\mathrm{R} 2 *}$, c) Linear regression between $\mathrm{LIC}_{\mathrm{SIR}}$ and $\mathrm{LIC}_{\mathrm{b}}$, and d) Bland-Altman plot of the difference vs. average of $\mathrm{LIC}_{\text {SIR }}$ in comparison to $\mathrm{LIC}_{\mathrm{b}}$

Figure 4: Mean of absolute differences between $\mathrm{LIC}_{\mathrm{b}}$ and $\mathrm{LIC}_{\mathrm{R} 2 *}$ or $\mathrm{LIC}_{\mathrm{SIR}}$ according to a) (Figure 5a) $\mathrm{LIC}_{\mathrm{b}}$ classes or b) (Figure 5b) $\mathrm{LIC}_{\mathrm{R} 2 * \text { classes }}$ 


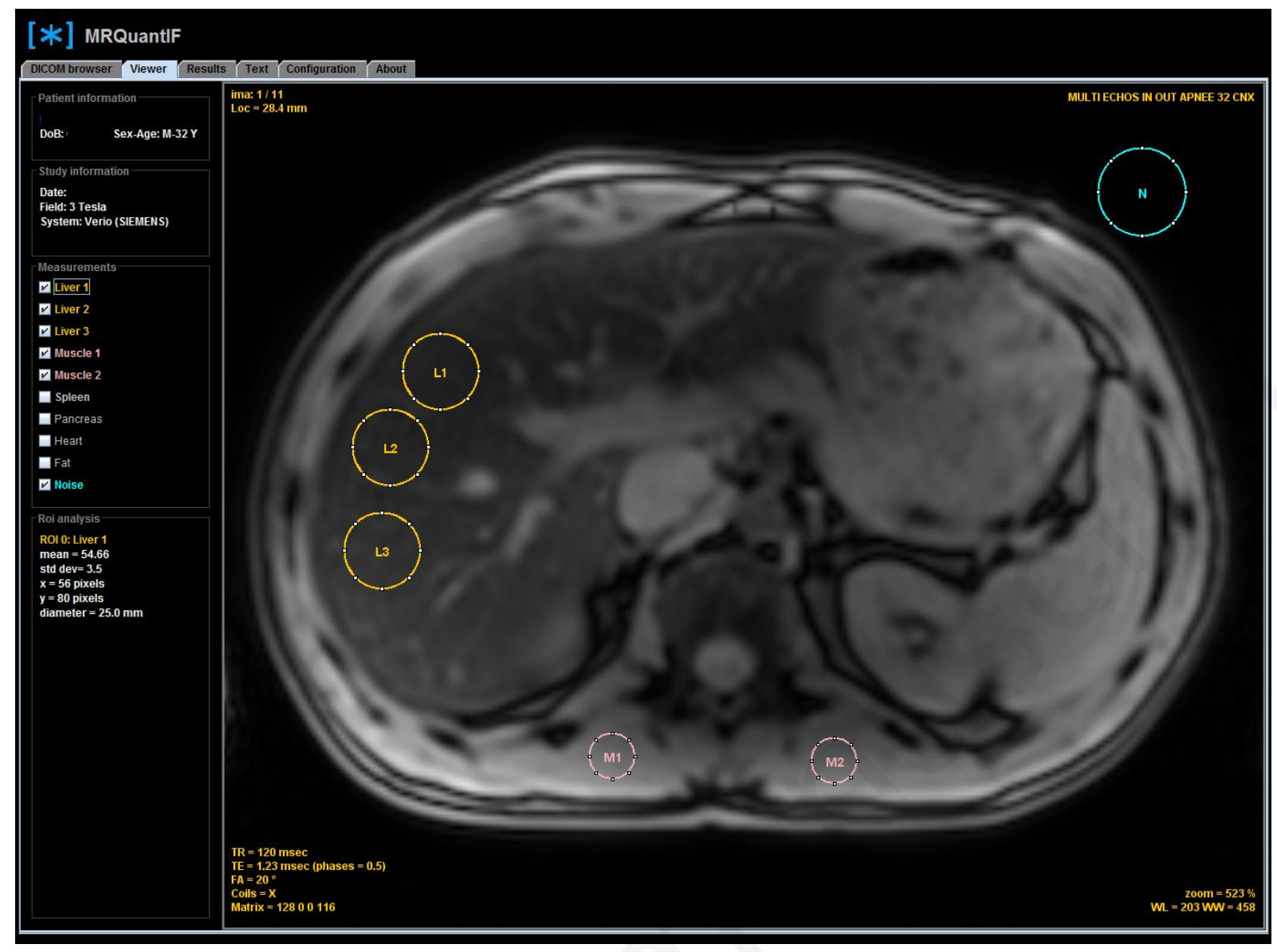

131 screened

3 without MRI

105 included 


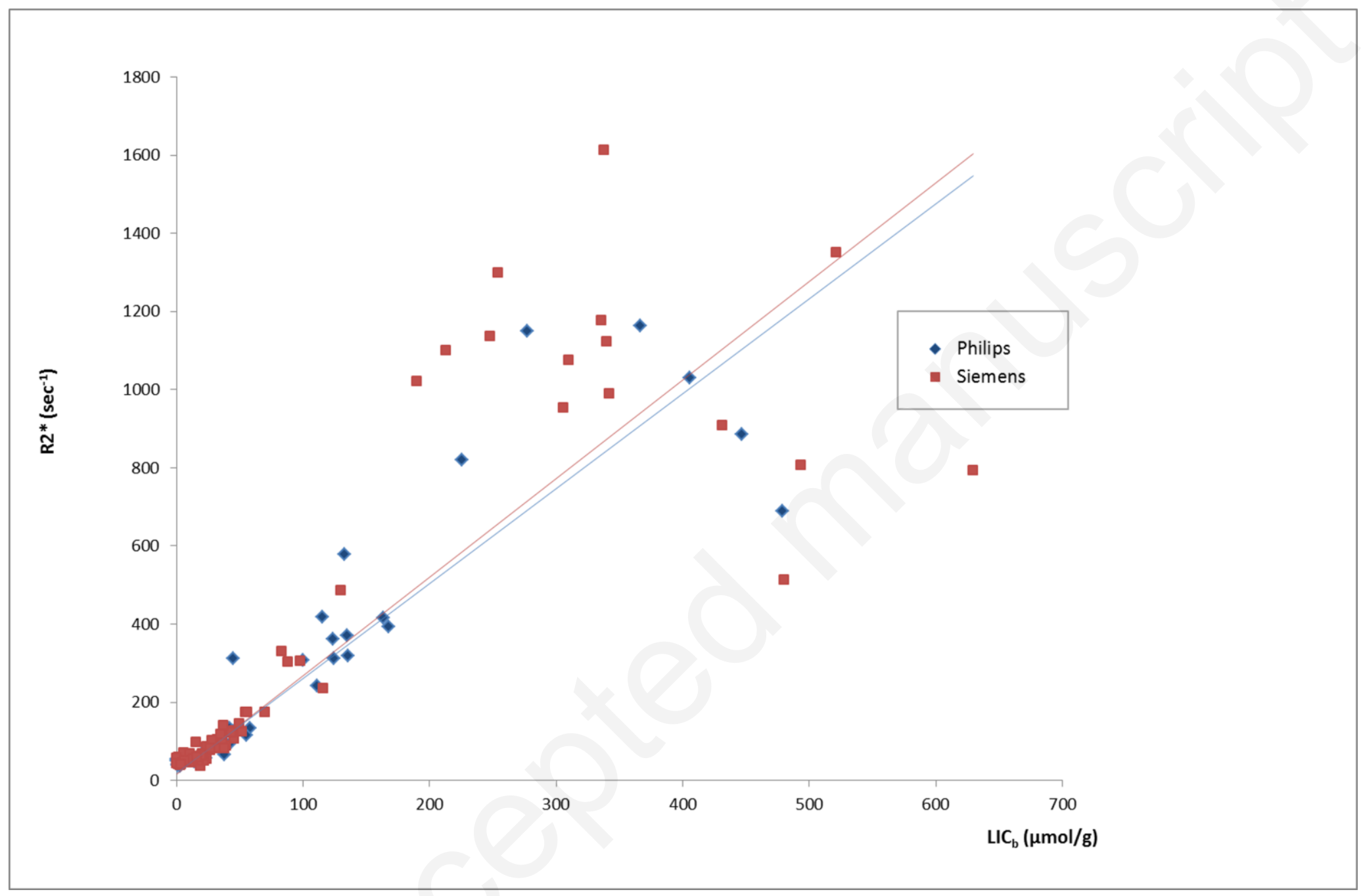

20 


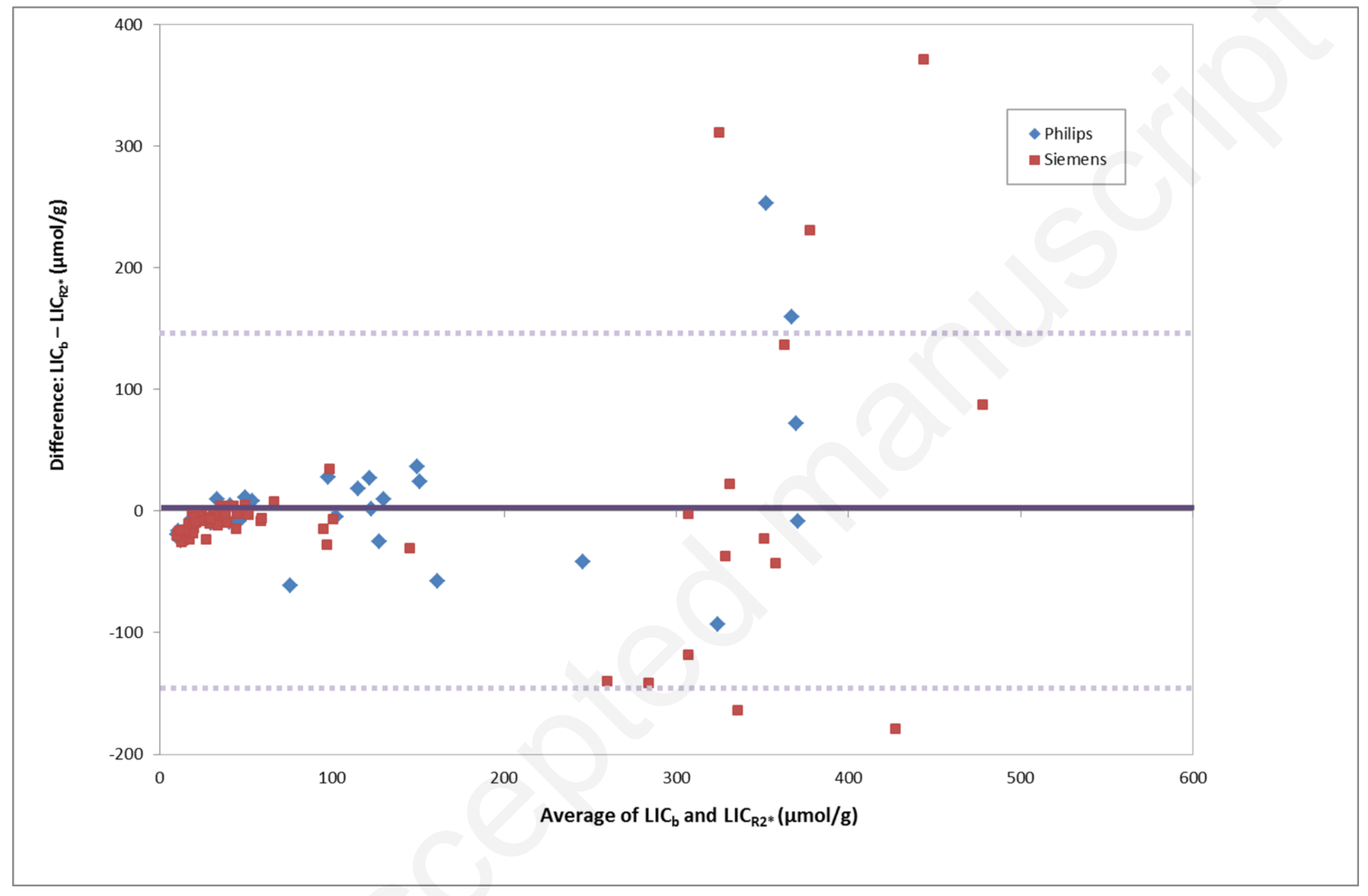




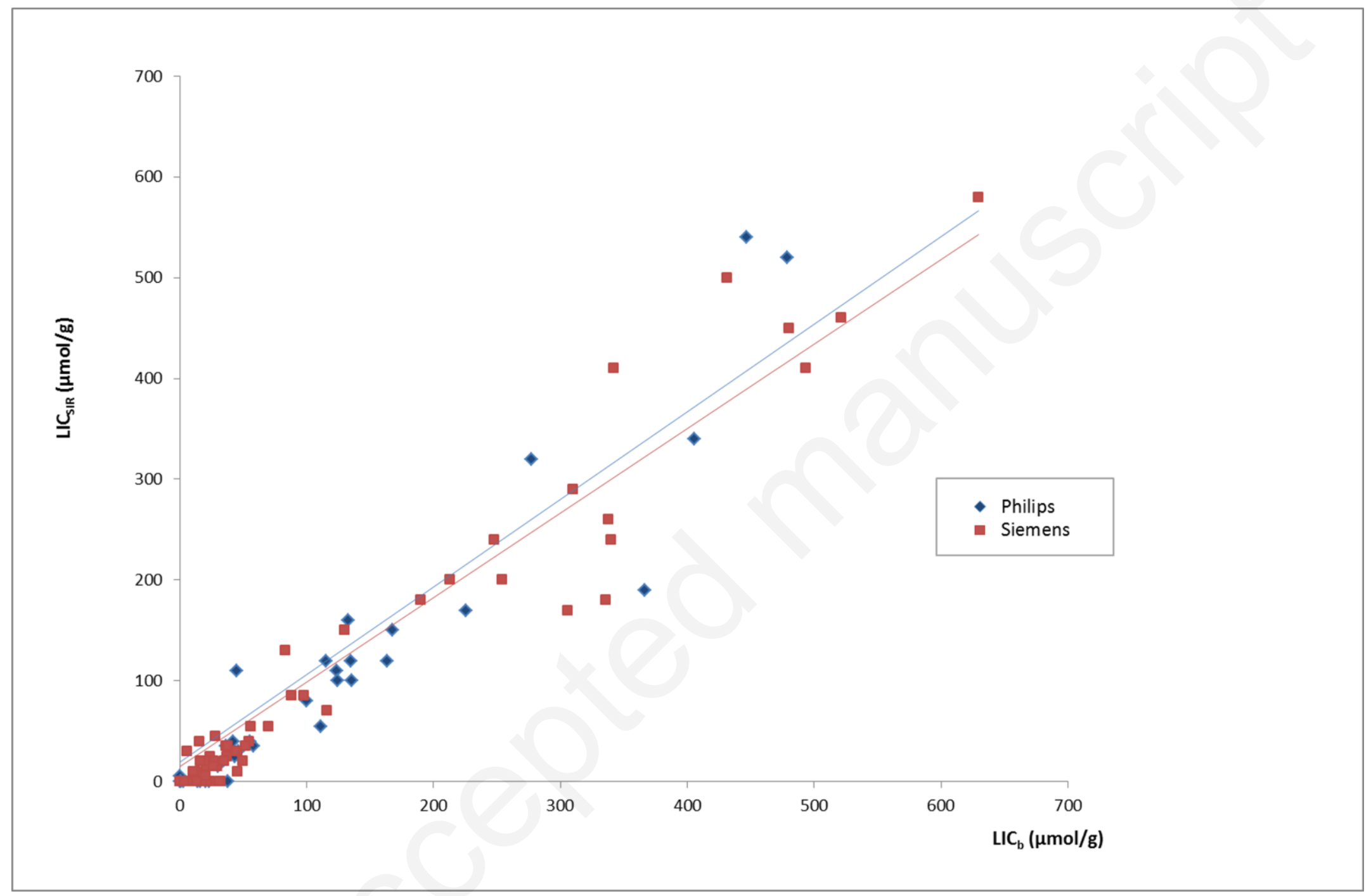




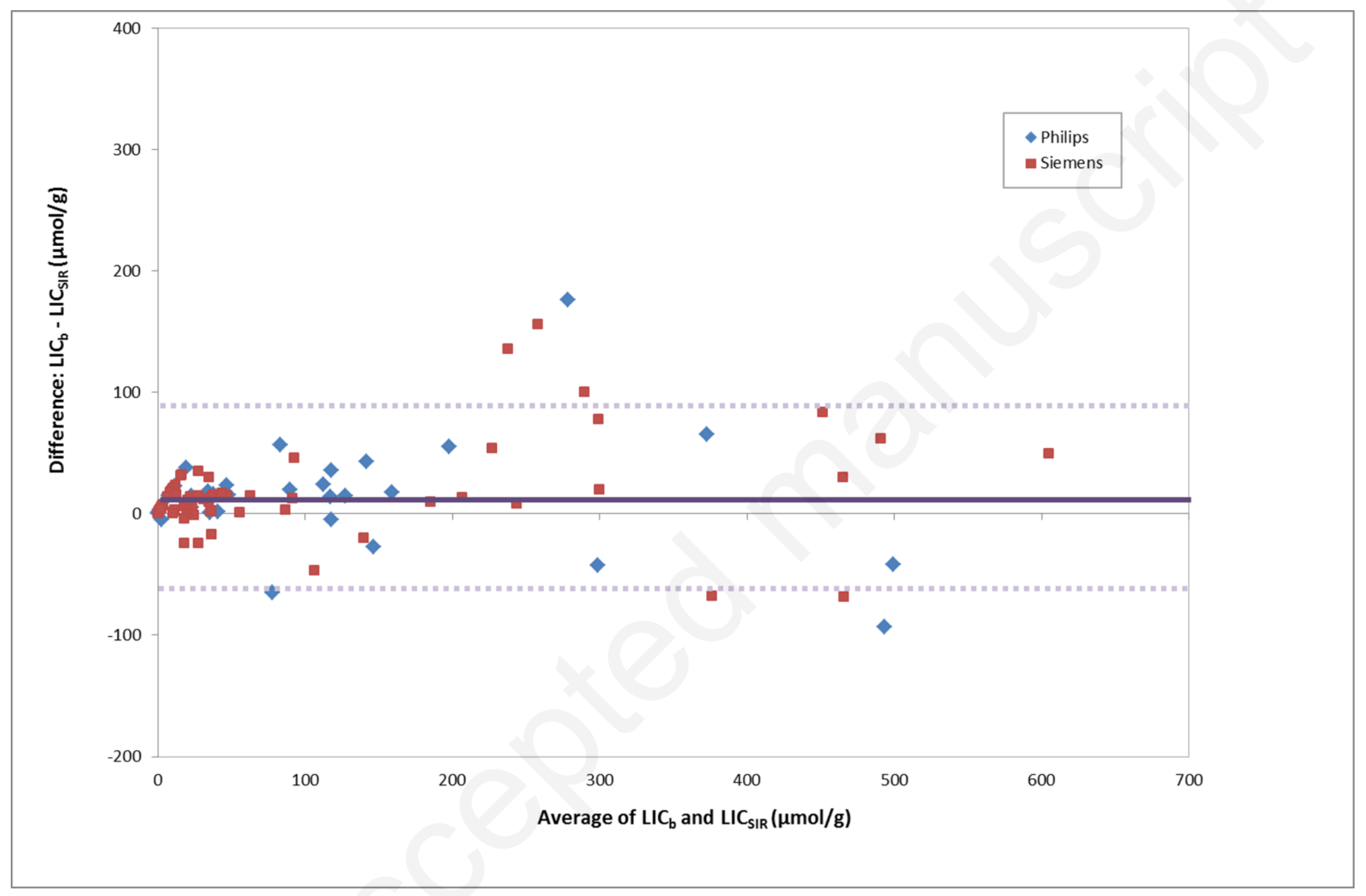




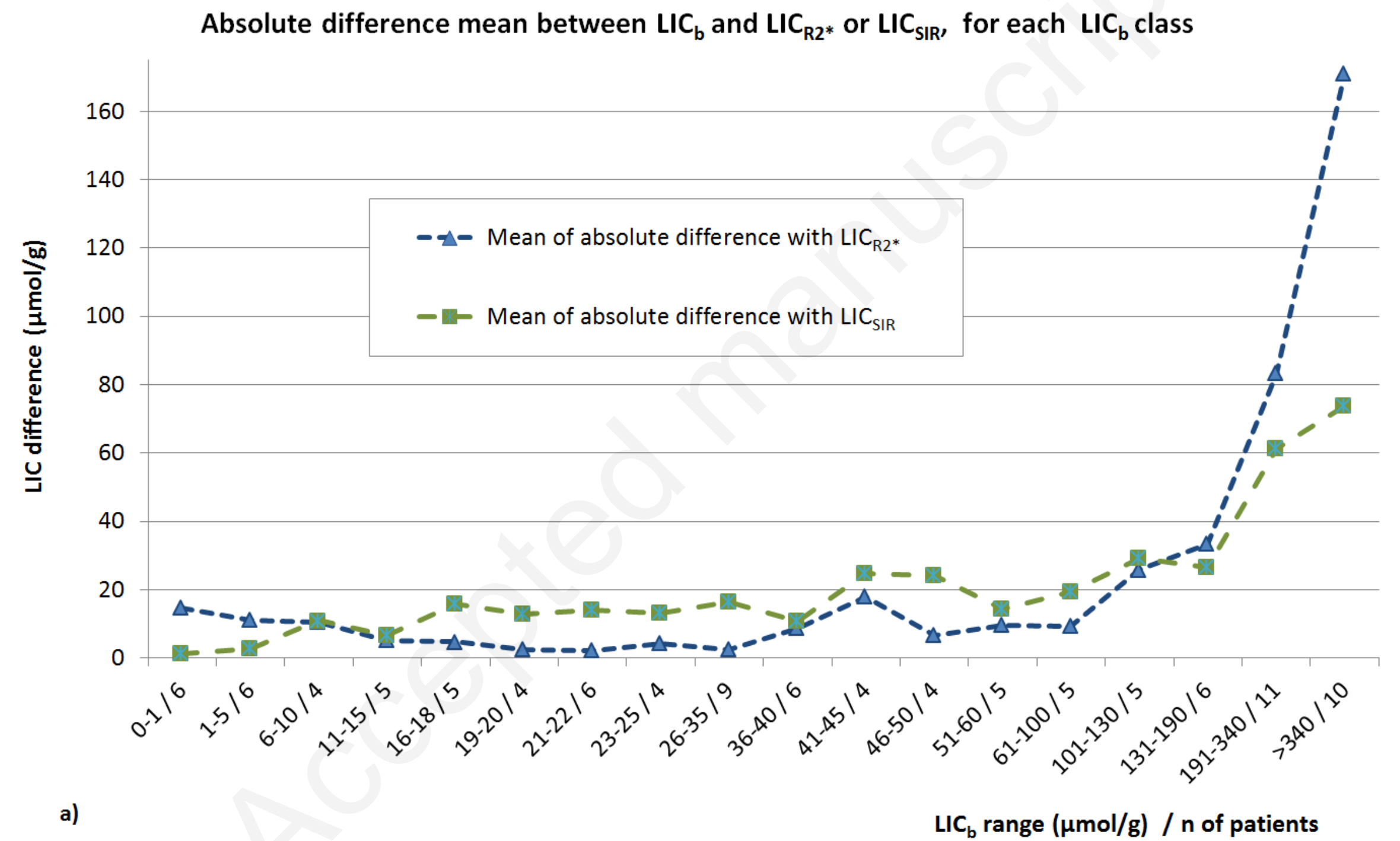




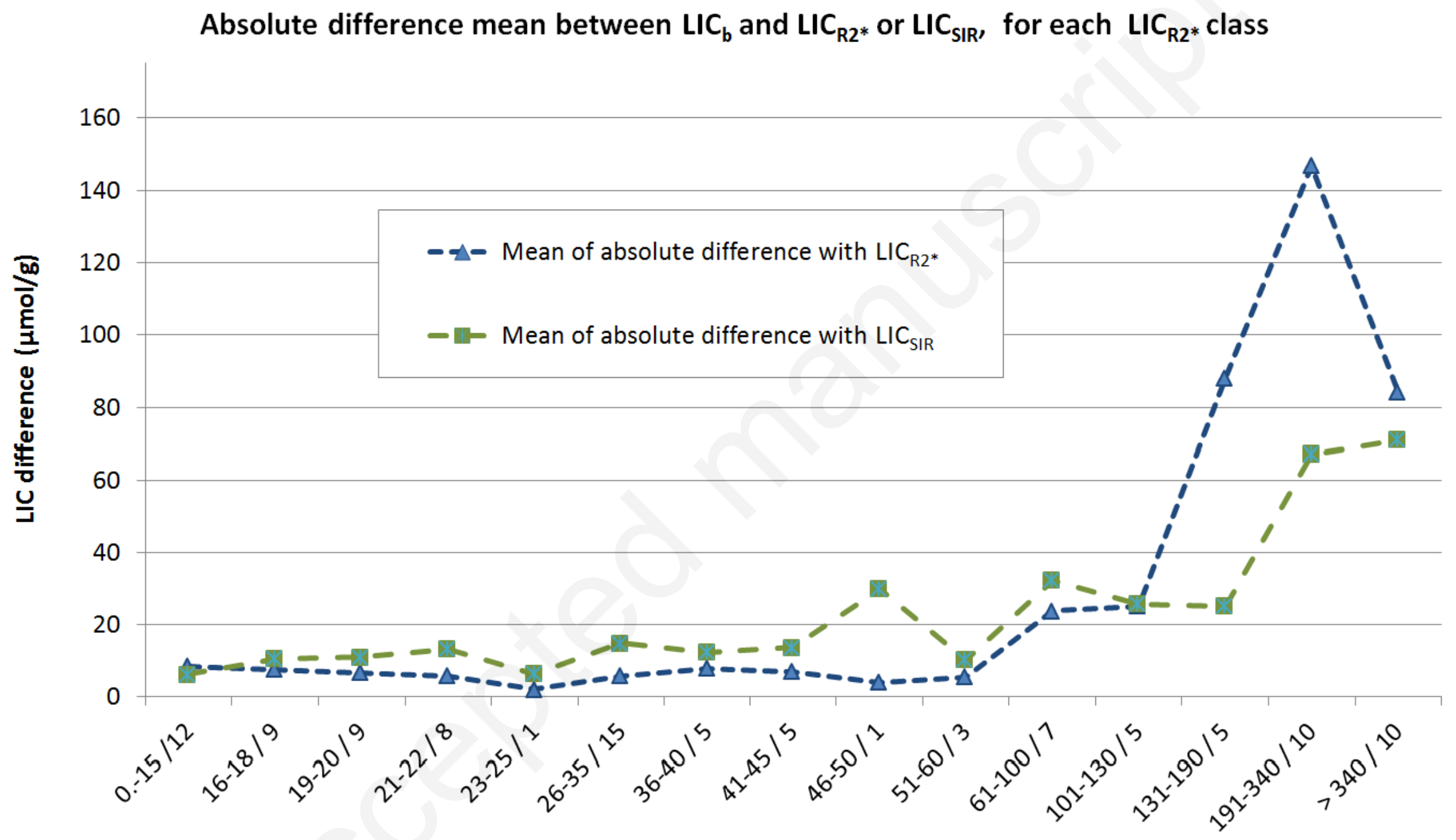

b)

$\mathrm{LIC}_{\mathrm{R}^{*}}$ range $(\mu \mathrm{mol} / \mathrm{g}) / \mathrm{n}$ of patients 